\title{
Testicular Metastasis of Colorectal Cancer: Case Report
}

Youness Khdach $^{1 *}$, Elias Andrea ${ }^{2}$, Raymond Andary ${ }^{3}$

${ }^{1}$ Military Hospital of Instruction Mohamed V, Hay Riad, 10100 Rabat, Morocco

${ }^{2}$ Service of Urology, Hospital Compiegne-Noyon, 8 Avenue Henri Adnot, 60200 ComPiègne, France

${ }^{3}$ Service of Urology, Hospital Compiegne-Noyon, 8 Avenue Henri Adnot, 60200 ComPiègne, France

DOI: $\underline{10.36347 / \text { sjmcr.2021.v09i02.010 }}$

| Received: 30.01.2021 | Accepted: 16.02.2021 | Published: 17.02.2021

*Corresponding author: Youness Khdach

Abstract

Metastatic carcinoma of the testis is rare and most often detected accidentally on pathological examination. The most common testicular metastases are secondary to prostate cancer (35\%), lung (18\%), melanoma (18\%), kidney (9\%) [1] while colorectal cancers represent less than $8 \%$ [2]. We report the clinical case of a 72-year-old patient, who presents abdominal pain with weight loss associated with a hydrocele that has progressed for 3 months with testicular discomfort. Exploration finds a sigmoid tumor with liver and bone metastases, scrotal ultrasound shows a vaginal nodule associated to hydrocele. An orchiectomy was performed with the anatomopathological result of two vaginal nodules responding to a very moderately differentiated, partially mucinous metastatic Lieberkuhnian adenocarcinoma. Through this clinical case and through a literature review, we analyze the epidemiological, diagnostic and therapeutic aspects of this rare pathology.

Keywords: Testicular Metastatic Colorectal Cancer, Metastatic carcinoma, clinical case.

Copyright $\odot 2021$ The Author(s): This is an open-access article distributed under the terms of the Creative Commons Attribution 4.0 International License (CC BY-NC 4.0) which permits unrestricted use, distribution, and reproduction in any medium for non-commercial use provided the original author and source are credited.

\section{INTRODUCTION}

Metastatic carcinoma of the testis is rare and most often detected accidentally on pathological examination. The most common testicular metastases are secondary to prostate cancer (35\%), lung (18\%), melanoma (18\%), kidney (9\%) [1] while colorectal cancers represent less than $8 \%$ [2]. Less than 15 cases have been published.

We report, in this article, a testicular metastasis of a sigmoid cancer discovered incidentally in a 72-year-old patient and analyze, through a review of the literature, the epidemiological, diagnostic and therapeutic aspects of this rare pathology.

\section{Patient and Observation}

Mr. O. S., 72 years old, diabetic with a history of cholecystectomy in 2016 without smoking, had consulted for abdominal pain since early July and loss of $6 \mathrm{~kg}$ in three months. An abdominopelvic ultrasound found secondary liver damage. A thoraco-abdominopelvic scan showed a sigmoid tumor with hepatic metastases and a strong suspicion of locoregional carcinoma with thrombosis of the right hepatic branch of the right branch of the portal trunk associated with a condensation of the left iliac of a suspicious appearance
(Figure 1). A rectosigmoidoscopy finds a tumor occupying more than two thirds of the circumference, of ulcerative aspect, located at the level of the sigmoid $(27 \mathrm{~cm}$ from the anal margin) and suggesting an adenocarcinoma. biopsy of this lesion finds a moderately differentiated sigmoid tubular ADK with a profile in favor of pMMR ADK (Mss, RER-). The search for somatic mutation of the KRAS, NRAS AND BRAF genes in colorectal $\mathrm{Kc}$ shows the presence of mutation of the KRAS gene conferring resistance to anti-EGFR Ab.

The patient was referred to us for management of a right hydrocele for 6 months with testicular discomfort. The scotal ultrasound finds normal looking testicles. The right testicle pushed back to the bottom of the scrotum by a heterogeneous fluid collection, partitioned with the presence of nodules Hyperechoic measured at $26 \mathrm{~mm}$, trilobed hypoechoic measured at $35 \mathrm{~mm}$, and simple hypoechoic measured at $10 \mathrm{~mm}$, hanging from the tunica vagina (Figure 2, Figure 3). The tumor markers were: AFP $1.7 \mathrm{ng} / \mathrm{ml}$, LDH / 1149 Ui / 1., HCG <2; ASAT: 91UI / L; ALAT 42UI / L; PAL 255 UI / L; GAMMA GT: 210 UI / L; CRP 119; ACE: $4853 \mu \mathrm{g} / \mathrm{l}$; CA 19-9: $18973 \mathrm{U} / \mathrm{mL}$. 
An upper route orchiectomy was performed (Figure 4) with the anatomopathological result of two vaginal nodules responding to a very moderately differentiated, partially mucinous metastatic lieberkuhnian adenocarcinoma and absence of neoplastic extension opposite the epididymal testicular pulp or cord and absence of vascular embolus (Figure 5, Figure 6).

\section{DISCUSSION}

Metastatic carcinoma of the testis is rare and most often detected accidentally on pathological examination. The most common tumors that metastasize to the testis are the prostate (35\%), lung (18\%), melanoma (18\%), kidney (9\%) [3] and colorectal less than $8 \%$ [3].

15 cases of colorectal cancer with metastases to the testis have been reported in the literature [3].

The exact mechanism of spread is unknown, but many theories have been suggested. Since most testicular metastases present as hydrocele, it is proposed that there may be microscopic communication channels between the peritoneum and the testes. Other theories include retrograde venous and lymphatic extension, direct invasion, and arterial embolism [4].

The primary lesion is mainly located in the ascending and transverse colon in $40 \%$ of cases, followed by the descending colon and sigmoid in $30 \%$ of cases and the rectum in the remaining 30\% [3]. The histological variant is adenocarcinoma, which constitutes 80 to $90 \%$ of cases [3].

If there is any doubt about a testicular tumor, the dosage of tumor markers (alpha-FP, beta-HCG) is always requested.

The scrotal ultrasound must be done in front of any hydrocele, it allows for the radiological diagnosis.

The treatment is based on the removal of the lesion by high orchiectomy. No action is justified on the contralateral testicle due to the rarity of bilateral involvement [2, 4]. Chemotherapy alone will not be as effective as the testes are considered a sanctuary site for chemotherapy [5].

The prognosis depends mainly on the presence of other metastases, as well as the radicality of the resection and the recurrence of peritoneal spread. Patients with testicular metastasis often carry highly disseminated disease at the time of diagnosis, with a poor prognosis.

\section{CONCLUSION}

Sigmoid cancer can be responsible for metastatic invasion of the testis. This damage is rare and poses the problem of etiological diagnosis. The high way orchiectomy is therefore essential. It allows histological confirmation of the diagnosis and eliminates another primary origin. The subsequent therapeutic attitude is part of the overall management of sigmoid cancer.

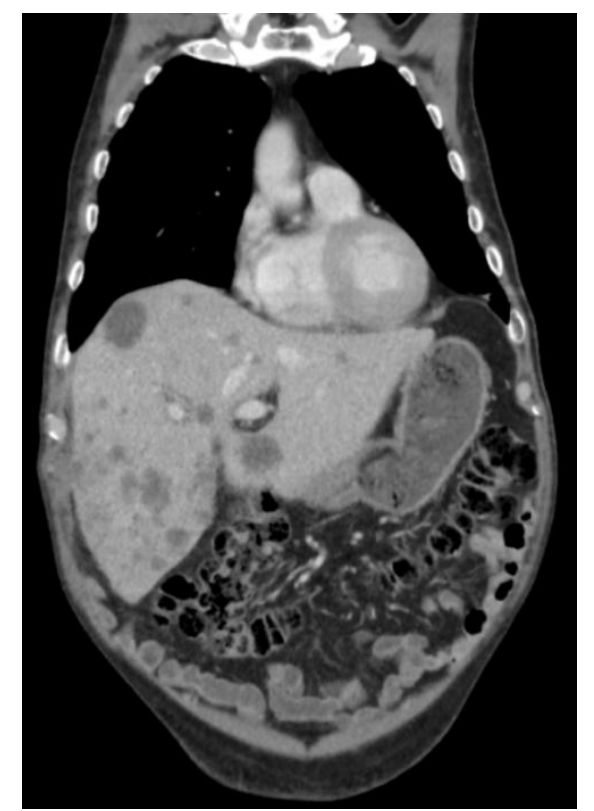

Figure 1: CT scan showing hepatic metastases

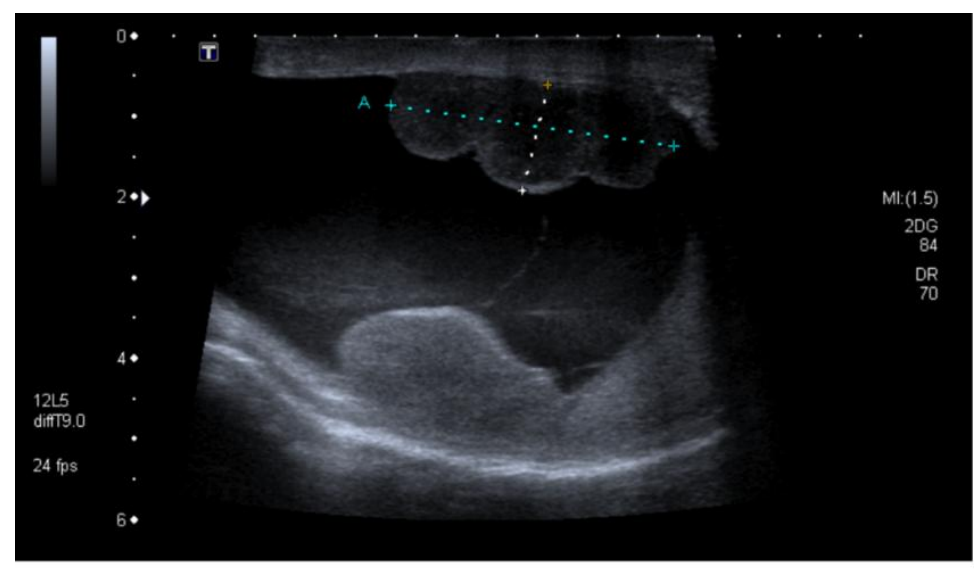

Figure 2 : Scotal ultrasound: vaginal nodule 


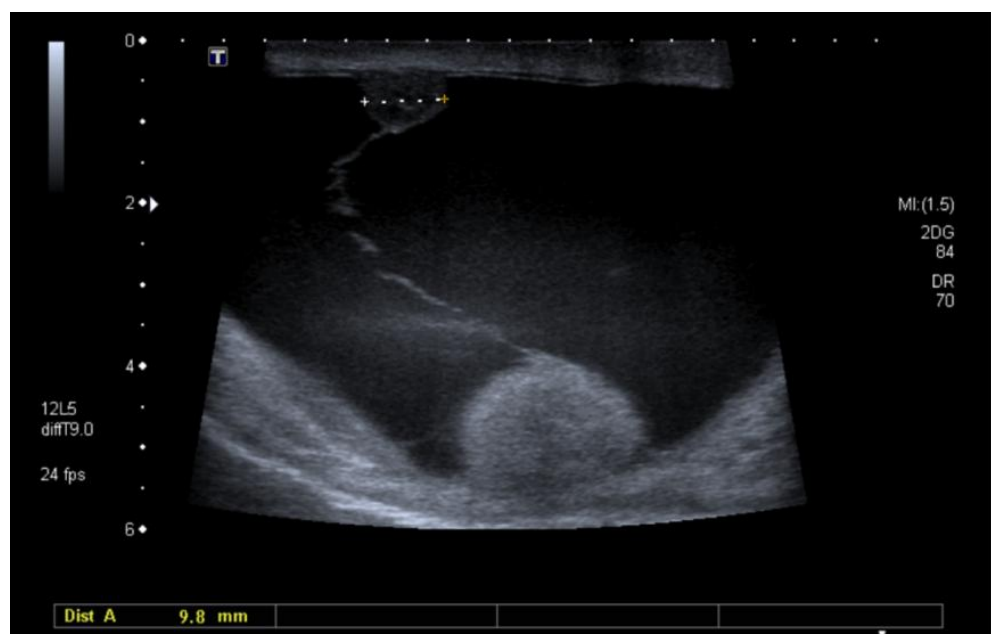

Figure 3 : Scotal ultrasound: vaginal nodule

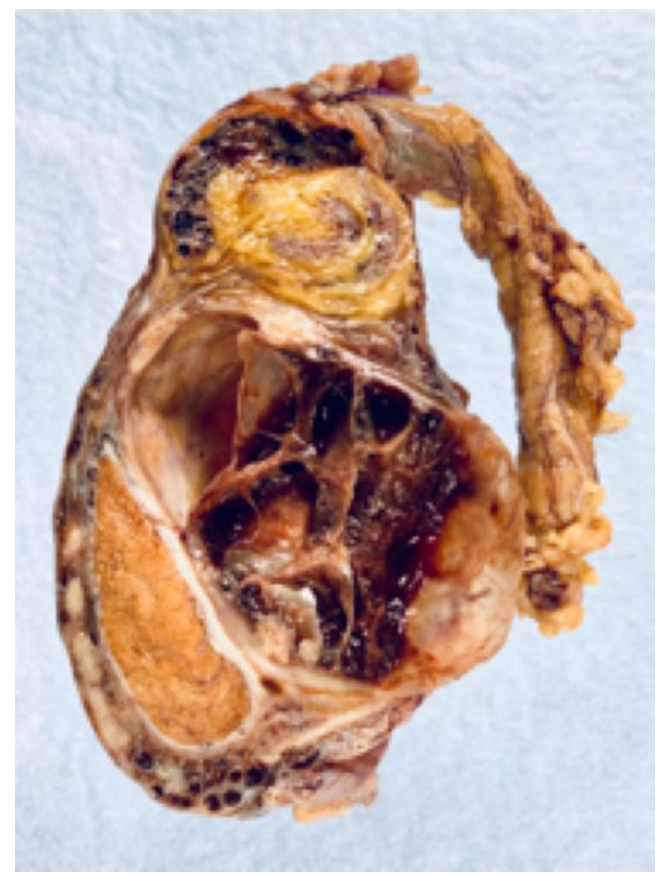

Figure 4 : Operative specimen of orchydectomy

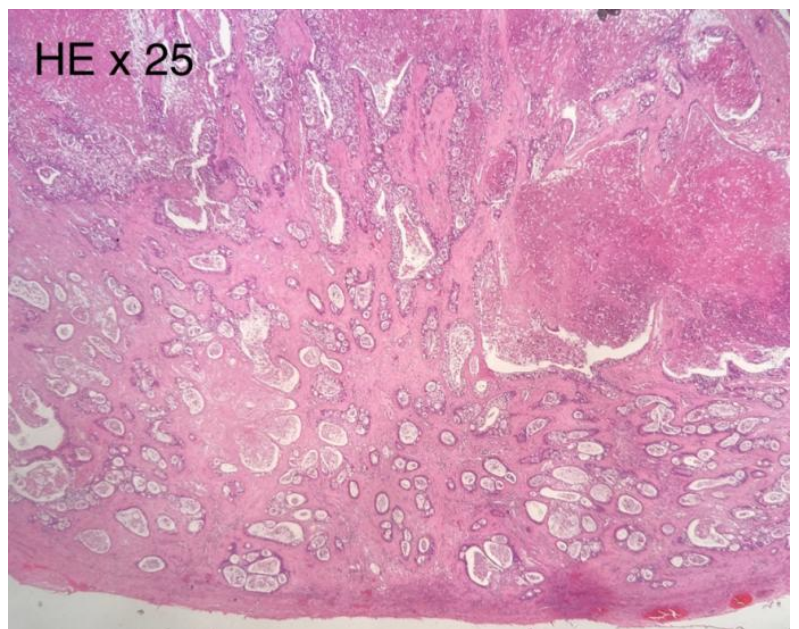

Figure 5 : Anatomopathology of Operative specimen: HE $\mathrm{X} 25$

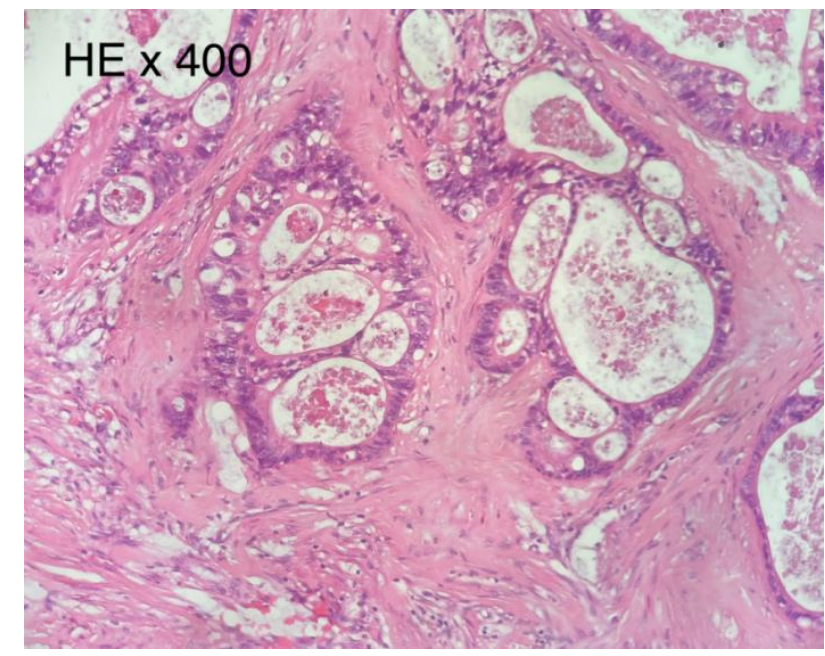

Figure 6 : Anatomopathology of Operative specimen: HE X 400

\section{REFERENCES}

1. Salesi N, Fabi A, Di Cocco B. Testis metastasis as an initial manifestation of an occult gastrointestinal cancer. Anticancer Res; 2004

2. Ulbright TM, Young RH. Metastatic carcinoma of the testis: a clinicopathologic analysis of 26 nonincidental cases with emphasis on deceptive features. Am J Surg Pathol

3. Navaroo M, Nicolas A, Ferrandez A, Lanas A. Colorectal cancer population screening programs worldwide in 2016: an update. World J Gastroenterol. 2017;23:3632-42.

4. Pratap Singh A, Kumar A, Dhar A, Agarwal S, Bhimaniya S. J Med Case Rep. 2018 Oct 11;12(1):

5. Smit JA, Baas JM, Verheijen PM. J Surg Case Rep. 2019 Oct 31;2019(10):eCollection 2019 Oct. 